\title{
Photosensitivity to piroxicam: absence of cross-reaction with tenoxicam
}

\author{
M. Gonçalo, A. Figueiredo, P. Tavares, C. A. Fontes Ribeiro, F. Teixeira and A. Poiares Baptista \\ Department of Dermatology and Institute of Pharmacology and Experimental Therapeutics, Faculty of \\ Medicine, University of Coimbra, 3000 Coimbra, Portugal
}

\begin{abstract}
We studied 2 groups of patients. One group of 10 patients had a photosensitive eruption to piroxicam. Another group of 24 patients had positive patch test reactions to thimerosal and thiosalicylic acid and had never taken piroxicam or tenoxicam. Patients were patch tested with thimerosal $0.1 \%$ pet., thiosalicylic acid $0.1 \%$ pet., salicylic acid $2.0 \%$ pet., piroxicam 1 and $5 \%$ pet. and tenoxicam 1 and $5 \%$ pet. Photopatch tests were also performed with piroxicam and tenoxicam. All 10 patients with photosensitivity to piroxicam had positive patch tests to thimerosal and thiosalicylic acid and 9 of them had positive photopatch tests to piroxicam. 20 out of 24 patients with positive patch tests to thiosalicylic acid also had positive photopatch tests to piroxicam. All the patients tested with salicylic acid were negative. Out of the 29 patients with positive photopatch tests to piroxicam, none reacted to tenoxicam. In countries with a high incidence of contact sensitivity to thimerosal/thiosalicylic acid, the use of piroxicam should be avoided and replaced by tenoxicam, a drug without reported photosensitivity.
\end{abstract}

Key words: piroxicam; tenoxicam; thimerosal; thiosalicylic acid; NSAIDs; photosensitivity; medicaments; lack of cross-sensitivity; chemical basis; allergen replacement.

Accepted for publication 7 April 1992

Piroxicam and tenoxicam are non-steroidal anti-inflammatory drugs (NSAIDs) of the 'oxicam' group of chemicals, unrelated to most of the commonly used non-steroidal anti-inflammatory agents. The former is one of the most frequently reported causes of drug-induced photosensitivity (1-5), whereas tenoxicam has not been reported as a cause of this adverse cutaneous reaction.

The pruritic erythemato-oedematous and vesicular lesions on sun-exposed skin, occurring in the course of systemic treatment with prioxicam, usually begin 1 to 2 days after the 1 st dose of the drug (3, 5-9). In most patients, lesions can be reproduced by a photopatch test with piroxicam at $0.5,1.0$ or $5.0 \%$ in petrolatum (pet.) irradiated with 5 to $10 \mathrm{~J} / \mathrm{cm}^{2}$ of UVA $(3,5,6,8-10)$.
Recently Serrano et al. (2) and De La Quadra et al. (10) described 5 patients with photocontact dermatitis from piroxicam. In 4 patients, the lesions developed within 2 days of a single application of piroxicam and none had previous exposure to this drug either topically or systemically.

Patients with systemic or contact photosensitivity to piroxicam have positive patch test reactions to thimerosal $(2,5,6,9,10)$ and, more precisely, to the thiosalicylate moiety of this molecule $(2,9,10)$. Furthermore, most of patients with contact sensitivity to thimerosal and thiosalicylic acid, and without previous exposure to piroxicam, have positive photopatch tests to this drug $(2,9,10)$ and can develop photosensitivity upon piroxicam ingestion $(9,10)$. 
We performed patch tests to salicylic acid and photopatch tests to tenoxicam in patients with photosensitivity to piroxicam or with contact sensitivity to thiosalicylic acid, in order to study their reactivity to this newer oxicam and to better understand the cross-reaction between piroxicam and thiosalicylic acid.

\section{Patients and Methods}

34 patients (19 male and 15 female) between 20 and 74 years old (mean 44.6 years) were studied. 10 patients ( 7 male and 3 female, with a mean age of 44.9 years) had a photosensitive eruption to piroxicam (group 1). 24 patients (12 male and 12 female, with a mean age of 44.5 years) had positive patch test reactions to thimerosal and thiosalicylic acid and had never taken piroxicam or tenoxicam (group 2).

A control group of 31 tested subjects (10 male and 21 female, with a mean age of 37.1 years) with negative patch test reactions to thimerosal and thiosalicylic acid was also studied (control).

Patients were patch tested with thimerosal $0.1 \%$ pet. (Trolab), salicylic acid (Merck) $2.0 \%$ pet. and thiosalicylic acid (Sigma) $0.1 \%$ pet. Readings were performed, according to the ICDRG guidelines, at 2 and 4 days. Duplicate patch tests with piroxicam (Pfizer) 1.0 and $5.0 \%$ pet. and tenoxicam (Roche) 1.0 and $5.0 \%$ pet. were applied on the back. Tests were removed after 2 days and then, while one set of tests was protected from light, the other was

Table 1. Patch and photopatch test results in patients with photosensitivity to piroxicam (group 1), patients with contact sensitivity to thimerosal/thiosalicylic acid (group 2) and controls.

\begin{tabular}{lcrc}
\hline & Group 1 & Group 2 & Control \\
\hline thimerosal & $10 / 10$ & $24 / 24$ & $0 / 31$ \\
thiosalicylic acid & $10 / 10$ & $24 / 24$ & $0 / 31$ \\
salicylic acid & $0 / 7$ & $0 / 13$ & $0 / 31$ \\
piroxicam (patch) & $0 / 10$ & $0 / 24$ & $0 / 31$ \\
tenoxicam (patch) & $0 / 10$ & $0 / 24$ & $0 / 31$ \\
piroxicam (photopatch) & $9 / 10$ & $20 / 24$ & $0 / 31$ \\
tenoxicam (photopatch) & $0 / 10$ & $0 / 24$ & $0 / 31$ \\
\hline
\end{tabular}

irradiated with UVA light from a PUVA unit (Psoralite, Paul B Elder Company, Ohio, USA) with $5.0 \mathrm{~J} / \mathrm{cm}^{2}$ of UVA. Readings were made just before irradiation and 2 days thereafter. Only ++ and +++ reactions were considered positive.

\section{Results}

As shown in Table 1, all 10 patients with photosensitivity to piroxicam (group 1) had positive patch tests to thimerosal and thiosalicylic acid, and 9 of them had positive photopatch tests to piroxicam. 7 of these 10 patients tested with salicylic acid were all negative.

20 out of 24 patients with positive patch tests to thiosalicylic acid (83.3\%) also had positive photopatch tests to piroxicam at 1.0 and $5.0 \%$ and none reacted to tenoxicam. 13 out of these 24 patients tested with salicylic acid were all negative (group 2).

Out of the 29 patients with positive photo-
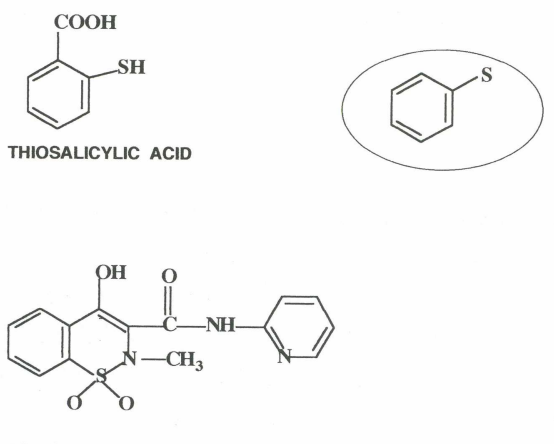

PIROXICAM

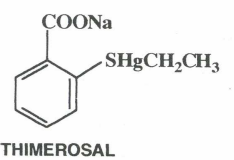

Fig. 1. Chemical structures of thimerosal, thiosalicylic acid and piroxicam. They have a common structural area composed of a benzene ring with an attached sulphur atom (in the case of piroxicam the attached sulphur atom is 'buried' within the adjoining ring): 
patch tests to piroxicam, none reacted to tenoxicam. No positive patch or photopatch test reactions to either piroxicam or tenoxicam were observed in the 31 control volunteers.

\section{Discussion}

Our studies, with a substantial number of patients, confirm that patients with photosensitive eruptions to piroxicam react to thimerosal and thiosalicylic acid in patch tests and to piroxicam in photopatch tests. $83.3 \%$ of our patients with positive patch tests to thiosalicylic acid also had positive photopatch tests to piroxicam, which is concordant with previous studies performed by De La Cuadra et al. (10), Serrano et al. (2), and Cirne de Castro et al. (9). Our work also shows that all patients reactive to thimerosal and thiosalicylic acid in patch, and to piroxicam in photopatch tests are negative to salicylic acid in patch, and to tenoxicam in photopatch tests.

When we examine the molecules to which these patients are reactive, we can identify a common structural area composed of a benzene ring with an attached sulphur atom (Fig. 1). These 2 structures are not present together either in salicylic acid or in tenoxicam (Fig. 2). This common molecular fraction is probably a constituent of the antigenic recognition area, which explains why these patients do not crossreact with salicylic acid and tenoxicam. These
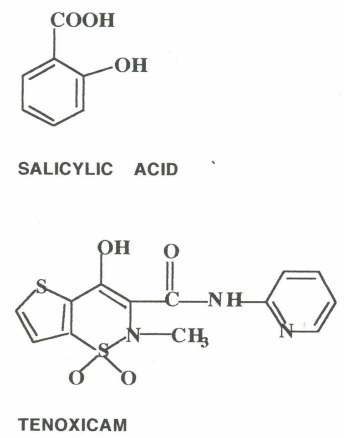

Fig. 2. Chemical structures of salicylic acid and tenoxicam. are important arguments to support the hypothesis of structural resemblance between thiosalicylic acid and piroxicam, first suggested by De La Quadra (10).

Until recently, we have accepted that piroxicam is stable to photolysis, even on prolonged exposure to light, based on our own work on nuclear magnetic resonance (3) and on studies performed by Pfizer Pharmaceuticals and communicated by Lombardino (11).

However, Miranda et al. (12) began to destroy this dogma by isolating and characterizing 2 photoproducts of piroxicam under aerobic conditions. Our group had recently shown in studies by HPLC with ultraviolet detection that piroxicam, like other non-steroidal antiinflammatory drugs, is photodegraded in vitro after UVA irradiation in a dose-dependent manner (13). Thus, it is possible that a photoproduct of piroxicam bears structural and/or antigenic resemblance to thiosalicylic acid and is responsible for the cross-reaction between these 2 drugs.

In order to test this hypothesis, we are now patch testing our patients with piroxicam photosensitivity with this drug irradiated in vitro. Piroxicam $0.25 \%$ in methanol, in nuclear magnetic resonance vials (with very thin glass and a large surface for irradiation), is irradiated with $50 \mathrm{~J} / \mathrm{cm}^{2}$ of UVA light from a PUVA unit. Our preliminary results show that 4 out of 7 patients with piroxicam photosensitivity have positive patch tests to this drug irradiated in vitro, which supports our previously reported hypothesis.

Theoretical and practical implications of all this knowledge are very interesting. For the first time, we can link an adverse effect of a systemic drug with an apparently dissimilar substance to which patients were previously exposed and to which they became sensitized. We can also deduce that the epidemiological characteristics of piroxicam photosensitivity are related to the use of thimerosal.

Thimerosal is a mercurial derivative extensively used as a preservative in inactivated vaccines, immunoglobulins and ophthalmic solu- 
tions and still employed in topical antiseptic solutions (Merthiolate tincture). The contact sensitization index to thimerosal is very variable throughout the world (14-20), from $1 \%$ in England (17) to $11.5 \%$ in Japan (20), but there are no pointers to know which of the components of thimerosal (ethylmercuric chloride or thiosalicylic acid) is responsible for sensitization. In our Department of Dermatology, the contact sensitization index to thiosalicylic acid in 688 patients tested in 1990 and 1991 was $6.4 \%$. This is a very high index and explains why photosensitive reactions to piroxicam are very frequent in our country and rare in other areas of the world.

In countries with a high incidence of contact sensitivity to thimerosal/thiosalicylic acid, the use of piroxicam should be avoided and replaced by tenoxicam, a drug with similar pharmacological characteristics, but without reported photosensitivity.

\section{References}

1. Stern R S, Bigby M. An expanded profile of cutaneous reactions to non-steroidal antiinflammatory drugs. Reports to a specialty based system for spontaneous reporting of adverse reactions to drugs. JAMA 1984: 252: 1433-1437.

2. Serrano G, Bonillo J, Aliaga A, Cuadra J, Pujol C, Pelufo C, Cervera P, Miranda M A. Piroxicam-induced photosensitivity and contact sensitivity to thiosalicylic acid. $J$ Am Acad Dermatol 1990: 23: 479-483.

3. Figueiredo A, Ribeiro C A F, Gonçalo S, Caldeira M M, Poiares-Baptista A, Teixeira F. Piroxicam-induced photosensitivity. Contact Dermatitis 1987: 17: 73-79.

4. Figueiredo A, Gonçalo M, Poiares Baptista A, Teixeira F. Reacçöes adversas medicamentosas em Dermatologia. Experiência do Núcleo de Farmacovigilância Dermatológica dos H.U.C. Coimbra Médica 1989: 10: 9-18.

5. McKerrow K J, Greig D E. Piroxicam-induced photosensitive dermatitis. J Am Acad Dermatol 1986: 15: 1237-1241.

6. Cirne de Castro J L, Vale E, Martins M. Mechanism of photosensitive reactions induced by piroxicam. J Am Acad Dermatol 1989: 20: 706.

7. Halasz C. Photosensitivity to the non-steroidal antiinflammatory drug piroxicam. Cutis 1987: 39: $37-39$.
8. Ljunggreen B. The piroxicam enigma. Photodermatol 1989: 6: 151-154.

9. Cirne de Castro J L, Freitas J P, Brandäo F M, Themido R. Sensitivity to thimerosal and photosensitivity to piroxicam. Contact Dermatitis 1991: 24: 187-192.

10. De La Cuadra J, Pujol C. Aliaga A. Clinical evidence of cross-sensitivity between thiosalicylic acid, a contact allergen, and piroxicam, a photoallergen. Contact Dermatitis 1989: 21: 349-351.

11. J G Lombardino, Pfizer Central Research, Groton C T, USA, personal communication, 1989.

12. Miranda M A, Vargas F, Serrano G. Photodegradation of piroxicam under aerobic conditions. The photochemical keys of the piroxicam enigma? J Photochem Photobiol B: Biol 1991: 8: 199-202.

13. Tavares $\mathrm{P}$, Teixeira $\mathbf{M}$ H, Figueiredo A, Fontes Ribeiro C A, Poiares Baptista A, Teixeira F. Photo-stability of non steroidal anti-inflammatory drugs. Proceedings IV Portuguese-Spanish Biochemistry Congress, Povoa de Varzim, 29 September- 2 October, 1991.

14. Hjorth N. Sensitivity to organic mercury compounds. Contact Dermatitis Newsletter 1967: 1: 15.

15. Rudner E J, Clendenning W E, Epstein E. Epidemiology of contact dermatitis in North America. Arch Dermatol 1973: 108: 537-540.

16. Tosti A, Guerra L, Bardazzi F. Hyposensitizing therapy with standard antigenic extracts: an important source of thimerosal sensitization. Contact Dermatitis 1989: 20: 173-176.

17. Cox N H, Forsyth A. Thiomersal allergy and vaccination reactions. Contact Dermatitis 1988: 18: 229-233.

18. Novak M, Kvicalova E. Analysis of the results of epicutaneous tests at the Dermatologic Clinic of Faculty Hospital in Prague. Cs Derm 1985: 60: 305-316.

19. Lachapelle J M, Chabeau G, Ducombs G, Lacroix P, Reuter L, Marot L. Enquête multicentrique relative à la frequence des testes épicutanées au mercure et au thimerosal. Ann Dermatol Venereol 1988: 115: 793-796.

20. Sato M. Patch test of mercury compounds and disinfectant solutions on clinical picture of mercury contact dermatitis. Jpn J Dermatol 1989: 99: 15-23.

Address:

Américo Figueiredo

Clinica de Dermatologia

Hospital da Universidade

3000 Coimbra

Portugal 